\title{
EU regulatory perspective on rare diseases: Focus on plasma protein related disorders
}

\section{Jacqueline Kerr}

Paul - Ehrlich - Institut, Paul Ehrlich Strasse 51-59, D-63225, Langen, Germany

E-mail: kerja@pei.de

This chapter on the EU regulatory perspective on rare plasma protein related diseases encompasses the following topics:

1. Regulatory Framework

2. Committee for Orphan Medicinal Products (COMP)

3. Rare diseases: Plasma Protein Related Disorders

\section{Regulatory framework}

Within the European regulatory framework for plasma related rare diseases the following topics should be considered:

- Legislation:

* Regulation (EC) No 141/2000 (on orphan medicinal products)

* Regulation (EC) No 847/2000 (provisions for implementation of orphan drug criteria)

* Regulation (EC) No 1902/2006 (paediatric regulation)

- Guidance Documents:

* Note for Guidance on Plasma-derived Medicinal Products

* Preclinical Safety Evaluation

* Guideline on Clinical Trials in Small Populations

* Core Summary of Product Characteristics (cSmPC) - product specific

* Note for Guidance (NfG) on the Clinical Investigation - product specific

\subsection{Legislation}

The Orphan Drug Regulation (EC) No 141/2000, proposed by the European Commission in July 1998, was adopted by the European Parliament and Council on 16 December 1999 and has been in force since 2000. It delineates the criteria for the Orphan Designation for diseases affecting less than 5 of every 10,000 patients in the EU and includes the following incentives: 
- Market exclusivity for up to 10 years,

- Fee reductions,

- Protocol assistance,

- Access to the Centralised Procedure for Marketing Authorisation.

In addition, the European Commission adopted the Commission Regulation (EC) No 847/2000 of 27 April 2000 which lays down the provisions for the implementation of the criteria for the Orphan Designation and defines "clinical superiority".

The main objective of the Paediatric Population Regulation (EC) No 1902/2006 (amending Regulation (EC) No 1901/2006) was to provide a legal framework in order to increase the availability of medicines specifically adapted and licensed for use in the paediatric population. This Regulation also applies to Orphan Medicinal Products and provides a two-year extension of market exclusivity, if information arising from a completed Paediatric Investigation Plan (PIP) is incorporated in the Summary of Product Characteristics (SmPC).

The above Regulations have been initiated to encourage companies in research, development and marketing of medicinal products to treat, prevent or diagnose rare diseases, and thus, to give patients suffering from these disorders access to the same quality of treatment as other patients.

\subsection{Guidance documents}

General and product specific guidance documents are available for quality, nonclinical and clinical development of plasma-derived products used in the treatment of rare diseases. The key guidance documents to be considered for development and marketing authorisation are listed below:

\subsubsection{Quality}

Note for Guidance on Plasma-Derived Medicinal Products (CPMP/BWP/ 269/95 rev.3)

The NfG covers all plasma-derived medicinal products and more specifically the source material (documented in a Plasma Master File (PMF)), the manufacture, the quality control and the validation studies, with special attention to viral inactivation/removal. Throughout this guideline it is assumed that the principles of Good Manufacturing Practise are followed and the requirements of the respective monographs of the European Pharmacopoeia are fulfilled.

\subsubsection{Non-clinical aspects}

Preclinical Safety Evaluation of Biotechnology-Derived Pharmaceuticals (ICH Topic S 6 - CPMP/ICH/302/95)

This guidance provides a basic framework for the preclinical safety evaluation of biotechnology-derived pharmaceuticals. However, the general principles on nonclinical testing strategy are also applicable to plasma derived products. 


\subsubsection{Clinical aspects}

Guideline on Clinical Trials in Small Populations (CHMP/EWP/ 83561/2005)

Since many rare diseases may affect only a few thousand or even less than one hundred patients in the EU, this Guideline has been developed to help tackle some of the problems associated with clinical trials in limited patient populations. Accordingly, conduct, analysis, and interpretation of studies in rare conditions at times may be constrained to varying degrees by the prevalence of the disease. Similarly, in paediatric trials it may be difficult to recruit sufficient numbers of patients. This document addresses general principles, which are by no means exhaustive but should encourage further exploration of potentially suitable methods for specific situations. In general, guidelines for trials in large populations are applicable to small populations. Any deviation from such established guidelines should be prospectively considered, and justified in the study protocol and further discussed in the study report. Seeking Scientific Advice, or Protocol Assistance, and expert statistical advice is strongly encouraged.

Product specific guidances and coreSPCs

- Core SPC for Human Prothrombin Complex Products (CPMP/BPWG/3735/02)

- Core SPC for Human Plasma Coagulation Factor VII Products (CPMP/BPWG/ 2048/01)

- Clinical Investigation of Plasma derived Antithrombin Products (CPMP/BPWG/ 2220/99)

- Core SPC for Human Plasma derived Antithrombin (CPMP/BPWG/3226/99)

- Clinical Investigation of Human Plasma Derived Factor VIII and IX Products (CPMP/BPWG/198/95)

- Clinical Investigation of Recombinant Factor VIII and IX Products (CPMP/ BPWG/1561/99)

- Core SPC for Human Plasma Derived and Recombinant Coagulation Factor VIII Products (CPMP/BPWG/1619/99)

- Core SPC for Human Plasma Derived and Recombinant Coagulation Factor IX Products (CPMP/BPWG/1625/99)

- Core SPC Human Normal Immunoglobulin for Intravenous Administration (IVIG), (CPMP/BPWG/859/95 rev. 2) (ongoing revision)

- Note for Guidance (NfG) on the Clinical Investigation of Human Normal Immunoglobulin for Intravenous Administration (IVIG) (CPMP/BPWG/388/95 rev.1) (ongoing revision)

These guidance documents can be found on EMEA website (www.emea.europa.eu/) and will be updated and revised to implement state-of-the-art regulatory and scientific development.

\section{Committee for Orphan Medicinal Products (COMP)}

An independent Committee for Orphan Medicinal Products (COMP) has been established at the European Medicines Evaluation Agency (EMEA), to review the 
eligibility of a given medicinal product with regard to an Orphan Drug Designation. The information on orphan drug designation is publicly available and provides patient access to a medicinal product (within a clinical trial) prior to marketing authorization.

\subsection{Regulatory routes}

There are several regulatory possibilities to obtain approval of a medicinal product with limited clinical data, e.g. by a marketing authorisation under exceptional circumstances, or a conditional marketing authorisation or compassionate use.

Exceptional Circumstances (Art. 14.8 Regulation No 726/2004)

In cases where comprehensive data cannot be submitted, a marketing authorisation under exceptional circumstances could be granted. This type of authorisation is reviewed annually to reassess the risk/benefit balance.

Conditional Marketing Authorisation (Art. 14.7 Regulation No 726/2004)

This type of application would apply in cases where the authorisation should be granted before all data are available, but it is expected that the outstanding information will be provided at a certain point in time in order to have a complete dossier.

Compassionate Use (Art. 83 Regulation No 726/2004)

A medicinal product can be made available for compassionate use reasons to patients with a chronic or seriously debilitating disease or whose disease is considered to be life-threatening, and who cannot be treated satisfactorily by an already authorised product. However, a product eligible for compassionate use needs either to be subject of a centralised application or must be undergoing clinical trials. The Committee for Medicinal Products for Human Use (CHMP) will adopt and regularly update the conditions for use and distribution.

\section{Rare diseases: Plasma Protein Related Disorders}

Plasma protein deficiencies are rare but often life-threatening diseases or disabilities such as primary immunodeficiencies, rare disorders treatable with immunoglobulins, haemostatic dysfunction and other disorders like alpha-1 proteinase inhibitor deficiency.

\subsection{Bleeding disorders and alpha-1 proteinase inhibitor deficiency}

\subsubsection{Factor VIII and Factor IX deficiency}

Haemostatic disorders comprise several hereditary dysfunctions that impair the body's ability to regulate blood coagulation. In its most common form, Hemophilia A, Factor VIII is absent, whilst in Hemophilia B, Factor IX is deficient. The prevalence of Hemophilia A is about 1 in 5,000-10,000, while Hemophilia B occurs at about 1 in 50,000. Regarding treatment of Hemophilia A and B several Factor VIII and IX products either plasma-derived or manufactured by recombinant technology are 
available to enable adequate patient supply in the EU. There are ambitious efforts ongoing in order to ameliorate patient compliance e.g. by prolongation of the half-life of Factor VIII to reduce treatment frequency.

\subsubsection{Factor VII deficiency}

Inherited factor VII deficiency is a rare autosomal recessive hemorrhagic disorder; the male-to-female ratio is $1: 1$. However, women are more likely to be symptomatic because of menorrhagia. Clinical bleeding can widely vary and does not always correlate with the level of FVII coagulant activity measured in plasma. The incidence is about 1:500 000 .

\subsubsection{Antithrombin deficiency}

Antithrombin is a potent inhibitor of the coagulation cascade. Congenital Antithrombin deficiency is an autosomal dominant disorder with a prevalence of 1:5000. This condition leads to increased risk of venous and arterial thrombosis, with an onset of clinical manifestations typically appearing in young adulthood.

\subsubsection{Protein $C$ deficiency}

Severe protein C deficiency occurs in approximately 1 in 500,000 to 1 in 750,000 live births. There are two main clinical features of severe Protein deficiency: skin necrosis when oral anticoagulant therapy is started and purpura fulminans in homozygous newborns.

\subsubsection{Other plasma protein deficiencies}

Examples for plasma protein deficiencies, where at present no specific products are on the EU market, are Factor V and X. In these very rare diseases treatment could be performed with fresh frozen plasma or prothrombin complex concentrates.

However, in those cases where plasma-derived products are available for rare plasma protein disorders in Europe, this does not mean that they are necessarily authorised throughout Europe. In some cases there are old marketing authorizations in only a few EU-countries, which would require some effort to update them to current requirements in order to support applications for marketing in other countries.

Due to the fact that bleeding disorders are mainly congenital diseases, aspects of the paediatric regulation are important to consider. On the one hand market launch for adults should not be hampered on the other hand children should be studied in clinical trials. Since the prevalence of most of these coagulation disorders is far below than 5:10 000, specific clinical guidance documents have been developed to reflect the particular clinical situation. It is important that the interests of patients to have swift access to products for rare diseases needs to be balanced by a careful efficacy and safety evaluation of each product. Recent revision of existing guidance documents (e.g. for FVIII and FIX) reflect these necessities as efficacy and safety has to be demonstrated in a smaller number of patients prior to marketing authorization when compared to post-marketing studies, which in turn have to be performed in a significant number of patients to show long-term compliance especially with regard to safety. 


\subsubsection{Alpha-1 Inhibitor deficiency}

Alpha1-Proteinase Inhibitor deficiency is a chronic, hereditary often fatal disorder, in which a low concentration of serum Alpha1-Proteinase Inhibitor is associated with progressive emphysema that most often manifests itself by the fourth decade of life. The deficiency occurs as a result of inheritance of two abnormal Alpha 1-Proteinase Inhibitor alleles from the Alpha1-Proteinase Inhibitor locus on chromosome 14. The discovery of this clinical association, together with the demonstration that enzymes with elastase activity experimentally induced emphysema when instilled into the lower respiratory tract of animals, have led to the "protease-anti-protease" concept of emphysema. This concept suggests that the alveolar structures of the lower respiratory tract in healthy individuals are protected from proteolytic attacks by proteases released by inflammatory cells through an anti-protease shield. The pathogenesis of emphysema in Alpha1-Proteinase Inhibitor deficiency is assumed to be a result of a chronic biochemical imbalance between elastase and its counteracting inhibitor, Alpha1-Proteinase Inhibitor. Alpha1-Proteinase Inhibitor deficiency is a rare disease with an estimated prevalence of 1:20-30 000 in the Caucasian population. Emphysema progression is a very slow process and commonly measured by the rate of annual decline in forced expiratory volume (FEV1). However, the quantitation of this decline on an individual basis is hampered by the high intra-individual variability of FEV1 measurements. Thus, clinical state-of-the-art studies are difficult to perform since a reliable clinical endpoint needs to be determined and a long term study period has to be covered.

\subsection{Rare immunological disorders}

The European regulatory framework and application procedures for intravenous human normal immunoglobulins (IVIG) have been outlined in a previous edition of Pharmaceuticals Policy and Law $(10,2008)$ whereby the main focus was on replacement therapy for Primary Immunodeficiencies (PIDs). The current edition will concentrate on rare disorders in the immunomodulatory setting. Rare disorders are those occurring in $<5 / 10.000$ persons. The auto-immune disorders addressed here are not "plasma-related" disorders in the classical sense; however, many exhibit abnormal auto-antibody production to various target organs or cells.

The revisions of both the IVIG core SPC and the Note for Guidance (NfG) which reflect the suggestions made at the EMEA Expert Meeting (June 2006), under the auspices of the Blood Products Working Party (BPWP) are currently ongoing and draft versions of these documents will be released for public consultation in February of this year (2009). The suggested revisions therefore are not final and will be subject to modification through the comments received by patient organizations, experts and industry.

For efficacy in the immunomodulatory setting Idiopathic Thrombocytopenic Purpura (ITP) is the cornerstone by which the immunomodulatory capacity of a given immunoglobulin product is confirmed. Although one disorder can obviously not 
encompass all facets and mechanisms of action of IVIGs, ITP offers some aspects that make it an attractive model. ITP is rare auto-immune disorder conveyed by known auto-antibodies to platelets (anti GPIIb/IIIa or/and Ib/Ix). The disorder can be studied in adults and children of both sexes. Platelets levels are easily measured and correspond to clinical manifestations (haemorrhages). After IVIG administration a response (i.e. rise in platelets to $>50 \times 10^{9} / \mathrm{L}$ ) occurs within $\sim 5$ days, this can be compared with other treatments or other IVIGs. The Fc-function of the IVIG has to be intact, as IVIGs with an impaired or modified Fc-region do not offer the same platelet response as non-modified IVIGs. Thus, this model is predictive of pathways that are IgG-Fc dependent.

If efficacy in ITP is adequately shown (response rate, duration, maximum platelet level and regression of haemorrhages) and corresponds to literature data, then no specific data are requested for Guillain Barré Syndrome (GBS) and Kawasaki's disease. $^{1}$

For other, currently "off-label" indications controlled clinical trials comparing the IVIG preparation with a placebo or with an established therapy are required to substantiate a marketing authorization. However, rare indications are by nature difficult to study esp. those disorders with an intermittent course. Depending on the availability and quality of the existing data, the extent of clinical data needed for a Marketing Authorization of a given IVIG product for a rare condition may vary. Study considerations should encompass whether other therapeutic modalities exist or have failed, if the disorder is potentially life threatening, if there are conflicting results in the literature and whether the pathomechanism (and subsequent administration of IVIG) is scientifically plausible. As mentioned above incentives and assistance provided by the Orphan Drug Regulation can be used by an applicant wishing to investigate a rare disorder. The Guideline on Small Populations (CHMP/EWP/83561/2005) should also be taken into consideration.

The following graph of current off-label uses encompasses indications found in the literature; various national Guidelines and review articles, but as there are $>150$ off-label uses, it does not profess to be comprehensive. It also does not include more common disorders $(>5: 10.000)$ such as multiple sclerosis, asthma, Alzheimer's disease or neonatal alloimmune thrombocytopenia, neonatal sepsis, where IVIG treatment may be beneficial.

In the revision of the Guideline and coreSPC the focus has been on neurological disorders of which the following were selected as "promising" indications due to the amount and quality of data available.

In general, the above graph is designed to give a flavour of the current IVIG setting and can be read in the following manner: the further to the front and the higher the indication columns are, the more likely a "regulatory approval threshold" will be reached by further well-designed studies with a given IVIG product.

\footnotetext{
${ }^{1}$ (Pending the final revision of the IVIG documents Allogeneic Bone Marrow Transplantation (aBMT) will in future most likely only be considered within the replacement setting when hypogammaglobulinaemia is present).
} 


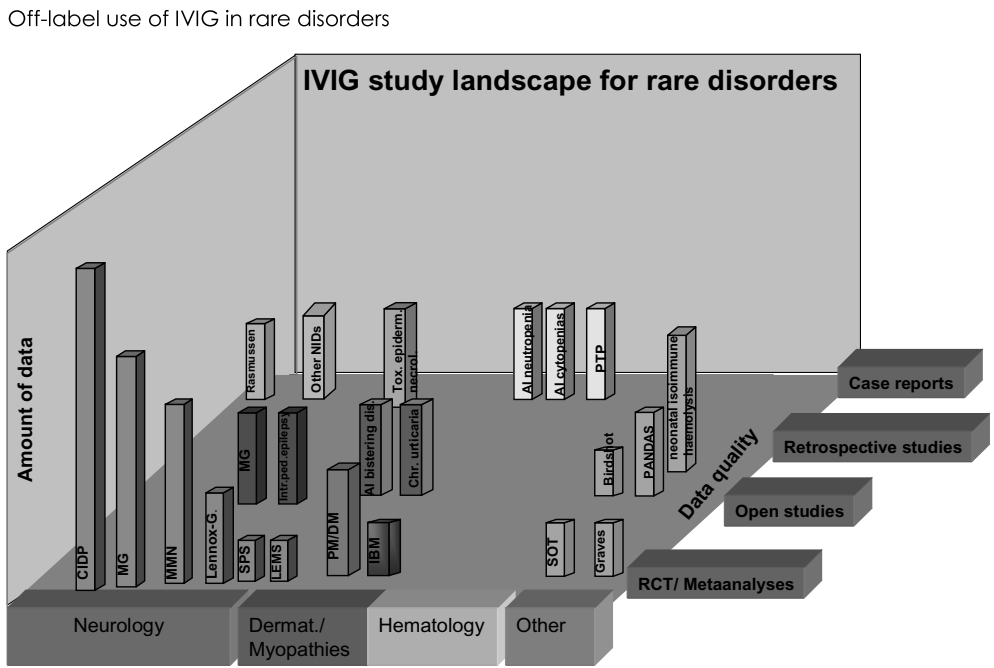

Fig. 1. Abbrev. from graph on IVIG study landscape. CIDP Chronic inflammatory demyelinating polyradiculoneuropathy; MMN multifocalmotor neuropathy; Lennox-Gastaut; MG Myasthenia Gravis; SPS Stiff-Person-Syndrome; LEMS Lambert-Eaton myasthenic syndrome; Intractable childhood epilepsy; Rasmussen syndrome; Other neuroimmune disorders (NIDs); PM/DM poly/dermatomyositis; IBM inclusion body myositis; AI blistering disorders; Chronic urticaria (delayed pressure); Toxic epidermal necrolysis/fasciitis; PTP posttransfusion purpura; AI neutropenia; Other AI cytopenias; SOT solid organ transplant (with humoral rejection); AI Graves ophthalmopathy; Birdshot retinochoroidopathy; PANDAS paediatric autoimmune neuropsychiatric disorders associated with streptococcal infections; Neonatal isoimmune $(\mathrm{ABO} / \mathrm{Rh})$ hemolysis.

\subsection{Chronic inflammatory demyelinating polyradiculoneuropathy (CIDP)}

CIDP is a rare neurological disorder with a prevalence of 1:100,000; and a preponderance of males $(\mathrm{m}: \mathrm{f}=2: 1)$. Generally the age of onset is at $\sim 50$ years, although the range covers a much wider span. The disorder can take a chronic progressive, relapsing remitting, or an acute course. The motor weakness which is more pronounced than any sensory loss, displays a symmetric pattern. Depending on the stage of the disorder muscle atrophy, motor conduction block and axonal loss can be part of the clinical presentation. In 6 randomised controlled trials (RCT) 4 different brands of IVIG were used. The treatment with IVIGs proved to be superior to placebo and equal to plasma exchange (PE) and prednisolone. Although the studies had some methodological flaws the overall consensus was that IVIGs had a place in the treatment CIDP. This has been confirmed by the positive results of the ICE study.

\subsection{Multifocal motor neuropathy $(M M N)$}

MMN is a chronic inflammatory condition with a prevalence $1: 100,000$, a male to female ratio of 2:1 and age of onset $\sim 40 \mathrm{y}$ (range 25-70). Anti-GM1 ganglioside 
antibodies of the IgM type can be found. The clinical presentation is one of motor weakness, muscle atrophy, fasciculations, cramps, whereby the weakness is more distally pronounced and more in the upper than the lower extremity. Motor conduction block and axonal loss are seen. In a Cochrane review (2005) of 4 RCT (34 pts.) with different brands of IVIG there was no statistically significant effect on disability $\left(1^{\circ}\right.$ endpoint of the review), however there was a significant effect on muscle strength ( $2^{\circ}$ endpoint) $(p=0.0005)$. Taking into consideration that corticosteroids and plasma exchange are not beneficial and cyclophosphamide is more toxic than IVIG, immunoglobulins may represent the safest treatment option.

\subsection{Myasthenia gravis $(M G)$}

MG is an autoimmune disorder $(2: 10,000)$, in which weakness is caused by circulating antibodies against acetylcholine receptors at the post-synaptic neuromuscular junction. The disorder can present as predominantly ocular or general. A myasthenic crisis occurs in $12 \%-16 \%$ of the patients and leads to respiratory paralysis necessitating assisted ventilation. Myasthenia is treated with cholinesterase inhibitors, plasma exchange, or immunosuppressants, and, in selected cases, thymectomy. Of the 5 RCTs, 3 were in patients experiencing exacerbations, 2 of which were adequately powered and one of these showed similar responses of plasma exchange and IVIG. Beneficial effects were confirmed in open and retrospective studies.

\section{Acknowledgement}

Any opinions/recommendations presented are our own and do not necessarily reflect those of the EMEA or the PEI. Our comments must not be interpreted as Guidance. 